\title{
¿Puede concursar como docente de la Facultad de Derecho de la Universidad de Buenos Aires una persona con discapacidad física para expresarse?
}

\section{POR ROMINA DEL VALLE ARAMBURU CÓRDOBA $(*)$}

\begin{abstract}
Sumario: I. Introducción.- II. ¿Qué es la discapacidad? - III. Cuestión cultural.- IV. Las normas jurídicas.- V. Normativas de la Universidad Nacional de Buenos Aires y de la Facultad de Derecho. "El derecho de enseñar". Perspectivas de implementación.- VI. Las eventuales limitaciones físicas para concursar.- VII. Posición a tomar por parte del ámbito académico.- VIII. ¿Es una cuestión que el ámbito académico debe resolver a instancia de parte? - IX. Cuando la promoción en la teoría y en la práctica de las igualdades pueden generar desigualdades.- X. Evitar las prácticas discriminatorias.- XI. Conclusión.- XII. Bibliografía.
\end{abstract}

Resumen: en el presente trabajo se tratará la posibilidad de que una persona quiera presentarse a concurso en el ámbito de la Facultad de Derecho de la Universidad de Buenos y ya tiene la discapacidad, pero tiene limitaciones físicas que le dificultan sobremanera la clase de oposición y el posterior dictado de las clases en caso de ganar el cargo para el que se postuló. Se realizará un análisis de cuáles son las eventuales limitaciones físicas para concursar y, en segundo lugar, en caso de ser seleccionado en un concurso de oposición y antecedentes, las posibilidades de poder dar clases desde un punto de vista fáctico; en ese caso, analizar cuál sería el rol del ámbito académico al respecto.

Palabras claves: discapacidad - concursos docentes - amparo - discriminación

Uma pessoa com deficiência físicas pode participar como professor da Faculdade de Direito da Universidade de Buenos Aires para se expressar?

Abstract: neste artigo discutiremos a possibilidade de uma pessoa querer participar de um concurso na área da Faculdade de Direito da Universidade de

$\left(^{*}\right)$ Procuradora, Abogada y Escribana. Esp. en Derecho Penal. Prof. Ordinaria Adjunta en Derecho Romano, Universidad Nacional de La Plata y Universidad de Buenos Aires (UNLP-UBA). Investigadora, Universidad Nacional de La Plata y Universidad de Buenos Aires. Autora del libro Instituciones de Derecho Romano, EDULP. 
Buenos e já ser portador de deficiência, mas apresentar limitações físicas que o tornam extremamente difícil para a turma de oposição e o Posterior Ditado das aulas em caso de conquista do cargo a que se candidatou. Será feita uma análise de quais são as possíveis limitações físicas para competir; e, em segundo lugar: no caso de ser selecionado em concurso de oposição e antecedentes, as possibilidades de poder dar aulas do ponto de vista factual, nesse caso é necessário analisar qual seria o papel do meio académico neste respeito.

Palavras chave: competições de ensino de deficiência - amparo - discriminação - incapacidade

\section{Introducción}

En el presente trabajo se va a tratar de determinar si hay posibilidades fácticas y jurídicas de que una persona con alguna clase de discapacidad física pueda concursar en la Facultad de Derecho de la Universidad de Buenos Aires. Para ello se hará referencia a la normativa vigente en la Universidad y su correlato en la Facultad. También en las normas nacionales e internacionales de Derechos Humanos. Luego se tratará de determinar cuál es el límite que debe tener para proceder institucionalmente en el caso de que una persona no se encuentre en condiciones físicas de poder desplegar la tarea docente y si la Facultad debe excluirla o proporcionarle otras herramientas para no incurrir en actos de discriminación.

\section{II. ¿Qué es la discapacidad?}

Según el Diccionario de la Real Academia Española, se vincula la misma a una situación de merma o carencia de alguna capacidad física, sensorial o psíquica de la persona, que limita o impide su participación plena e igualitaria en la sociedad o el ejercicio efectivo de sus derechos(1).

Para la Organización Mundial de la Salud es un término general que abarca las deficiencias, las limitaciones de la actividad y las restricciones de la participación. Las deficiencias son problemas que afectan a una estructura o función corporal; las limitaciones de la actividad son dificultades para ejecutar acciones o tareas, y las restricciones de la participación son problemas para participar en situaciones vitales.

Por consiguiente, la discapacidad es un fenómeno complejo que refleja una interacción entre las características del organismo humano y las características de la

(1) Recuperado de https://www.google.com/search?ei=csw5X7XwOImz5OUPxfinuAM\&q=conc epto+de+discapacidad+segun+la+real+academia+espa\%C3\%B1ola 
¿Puede Concursar como docente de la Facultad de Derecho de la Universidad DE BUENOS AIRES UNA PERSONA CON DISCAPACIDAD FÍSICA PARA EXPRESARSE?

- Romina Del VAlle ARAMburu CóRdoba (PP. 347-366)

sociedad en la que vive. Sin embargo, no se trata de una definición pétrea. Es flexible desde las ópticas que sean necesarias analizar: La Clasificación Internacional del Funcionamiento, de la Discapacidad y de la Salud (CIF) define la discapacidad como un término genérico que abarca deficiencias, limitaciones de la actividad y restricciones a la participación. Se entiende por discapacidad la interacción entre las personas que padecen alguna enfermedad (por ejemplo, parálisis cerebral, síndrome de Down y depresión) y factores personales y ambientales (por ejemplo, actitudes negativas, transporte y edificios públicos inaccesibles y un apoyo social limitado).

En la Convención sobre los Derechos de las Personas con Discapacidad, que fue aprobada en la Asamblea General de las Naciones Unidas en el año 2006, plantea un concepto amplio, que puede dar lugar a incertidumbre en algunos casos. En su artículo 1, párrafo segundo, enuncia a quienes se pueden considerar personas con discapacidad: quedan incluidas aquellas con deficiencias físicas, mentales, intelectuales o sensoriales a largo plazo que, al interactuar con diversas barreras puedan impedir su participación plena y efectiva en la sociedad, en igualdad de condiciones con las demás (Seda, 2015, p. 11).

Se ha reelaborado un nuevo concepto de discapacidad, según las transformaciones culturales, y no está vinculado solo a enfermedades visibles sino a dolencias de toda clase, incluso psicológicas. A lo largo del tiempo se han utilizado términos como discapacidad, capacidades diferentes o "se alude al fenómeno como sinónimo de no-capacidad y por ende a personas no capaces, con la intención de eliminar connotaciones peyorativas y negar discursivamente $-\mathrm{y}$ de manera falaz- la cualidad de no capaces que se atribuye naturalmente a esta población" (Mareño Sempertegui, 2012, p. 139).

El Programa de Acción Mundial para los Impedidos (sic) (Naciones Unidas, 1982) representó un avance al señalar que la minusvalía es una desventaja creada por la sociedad que no se origina en el problema de una persona, sino en la ausencia de oportunidades; ocurre cuando dichas personas se enfrentan a barreras culturales, físicas o sociales que les impiden el acceso a los diversos sistemas de la sociedad que están a disposición de los demás ciudadanos.Laminusvalíaes, portanto,lapérdidaolalimitacióndelasoportunidades de participar en la vida de la comunidad en igualdad con los demás.

En la Clasificación Internacional del Funcionamiento, de la Discapacidad y de la Salud (Naciones Unidas, 2001) se abarcan tres dimensiones: 1) funciones y estructuras corporales; 2) actividad; 3) participación. Se identifican para las mismas las limitaciones que pueden presentar: deficiencias, limitaciones en la actividad, restricción en la participación. Se habla entonces de deficiencias de la función y 
deficiencias de la estructura, actividades (antes discapacidades) y participación (antes minusvalía) respectivamente (Dell'Anno, 2012, p. 13).

Al hacer referencia a la discapacidad, tenemos que concientizar en la teoría y en la práctica diaria acerca de la inclusión; este término ni puede faltar en el tratamiento de esta temática. Inclusión como una temática teórico y práctica vinculadas a la cooperación, vinculación, proyecciones en búsqueda de soluciones para las personas que atraviesan por estas cuestiones.

Se debe tener en cuenta tanto al sujeto como persona como así también a su contexto como integrante de una comunidad, de un todo. "Las personas con discapacidad (...) también son considerados sujetos de las políticas públicas" (Valiente Noailles, 2017, p. 126).

\section{Cuestión cultural}

Históricamente, las personas con discapacidad fueron segregadas de la sociedad. Si nos remontamos a la historia de la humanidad, por ejemplo, el pueblo romano, desde la fundación de Roma en el año 759 a.C., a los sujetos que tenían malformaciones graves los arrojaban por la roca Tarpeya (un precipicio). Se los tenía como que nunca habían tenido trascendencia como persona. Para entender estas cuestiones había que entender la idiosincrasia romana. Hoy no lo aceptamos porque las sociedades evolucionaron en otro sentido y, además, culturalmente nos regimos por otros parámetros: la protección de las personas con discapacidad.

En nuestro país se observa una larga tradición de tener a las personas con discapacidad, en cierta medida, relegadas de ciertas participaciones de la vida social o laboral, por eso la necesidad de su inclusión por el cupo laboral. Las personas con limitaciones físicas - son objeto de análisis no menor los casos de limitaciones mentales en las que los sujetos pueden desempeñar una actividad laboral, pero hay que analizar cada caso en particular- pueden llevar a cabo diversas actividades adecuándose a esas limitaciones.

La accesibilidad se entiende como la posibilidad de las personas con movilidad reducida de gozar de las adecuadas condiciones de seguridad y autonomía como elemento primordial para el desarrollo de las actividades de la vida diaria, sin restricciones derivadas del ámbito físico urbano, arquitectónico o del transporte, para su integración y equiparación de oportunidades - consagrado por el artículo 20 de la ley 24.314-.

En los tiempos actuales, lo primero que se tiene que propiciar es la toma de conciencia, no solo de la comunidad académica, sino de toda la sociedad sobre estas cuestiones. Hay que transformar ciertos parámetros sociales o culturales y 
¿Puede Concursar como docente de la Facultad de Derecho de la Universidad DE BUENOS AIRES UNA PERSONA CON DISCAPACIDAD FÍSICA PARA EXPRESARSE?

- Romina Del VAlle ARAMburu CóRdoba (PP. 347-366)

erradicar la postura simplista de excluir al que tiene dificultades, o que el propio sujeto se autoexcluya.

Lo segundo, es la voluntad política de llevar adelante políticas públicas en este sentido.

\section{Las normas jurídicas}

\section{IV.1. Normas nacionales e internacionales}

El artículo 16 de la Constitución Nacional Argentina establece en el consagrado principio de igualdad ante la ley que “(...) Todos sus habitantes son iguales ante la ley, y admisibles en los empleos sin otra condición que la idoneidad (...)”.

Lo primero que hay que analizar son los carriles legales, antes de llegar a los medios en sí mismo para poder concursar. En principio, el artículo 16 de la Constitución Nacional, como ya se dijo, consagra la igualdad de las personas, pero la igualdad de oportunidades será en la medida que haya igualdad de condiciones. La Corte Suprema de Justicia de la Nación ha establecido:

El principio de la igualdad de todas las personas ante la ley, según la ciencia y el espíritu de nuestra Constitución, no es otra cosa que el derecho a que no se establezcan excepciones o privilegios que excluyan a unos de lo que se concede a otros en iguales circunstancias, de donde se sigue forzosamente que la verdadera igualdad consiste en aplicar en los casos ocurrentes la ley según las diferencias constitutivas de ellos y que cualquiera otra inteligencia o acepción de este derecho es contraria a su propia naturaleza e interés social (Fallos: 16:118; 123:106; 124:122).

Aquí, a la vez, se van presentando otras cuestiones: la primera igualdad es el derecho a concursar si se cumplen con los requisitos administrativos. El segundo plano de igualdad está en que cada concursante pueda hacer su ponencia de conformidad a lo establecido por el reglamento, pero: ¿Qué sucede cuando hay desigualdades por causas físicas? ¿Ello justifica que un ámbito académico haga una erogación para no dejar a fuera al postulante por sus limitaciones físicas? Si no hay una normativa que justifique que se pueda destinar presupuesto a dicha cuestión, parece ser muy difícil. Entonces, si se da esto último, ¿estamos excluyendo a un eventual postulante? Tenemos que tener en cuenta que "Actualmente, se asiste a un movimiento que puede considerarse cultural y político, donde se observan iniciativas de las personas con discapacidad, tendientes a reafirmar su autonomía 
e independencia, como personas adultas y dándose sus propias orientaciones" (Dell' Anno, 2012, p. 15).

La Convención Interamericana para la Eliminación de Todas las Formas de Discriminación contra las Personas con Discapacidad (OEA, 1999), que fue aprobada en Argentina por ley 25.280 (2000), en sus considerandos expresa que: "Las personas con discapacidad tienen los mismos derechos humanos y libertades fundamentales que otras personas; y que estos derechos, incluido el de no verse sometidos a discriminación fundamentada en la discapacidad, dimanan de la dignidad y la igualdad que son inherentes a todo ser humano"; luego agrega: "Considerando que la Carta de la Organización de los Estados Americanos (...) establece como principio que la justicia y la seguridad sociales son bases de una paz duradera". También podemos observar en la Convención una serie de recomendaciones específicas destinadas a disminuir los riesgos de dicha discriminación (Dell' Anno, 2012, p. 14).

La normativa no se agota allí. Podemos mencionar la Convención Internacional de Derechos Humanos de las Personas con Discapacidad (2006), la cual en su artículo 3 consagra los principios generales, entre ellos: el respeto de la dignidad inherente, la autonomía individual, incluida la libertad de tomar las propias decisiones y la independencia de las personas; la no discriminación; la participación e inclusión plenas y efectivas en la sociedad; el respeto por la diferencia y la aceptación de las personas con discapacidad como parte de la diversidad y la condición humanas; la igualdad de oportunidades; la accesibilidad; la igualdad entre el hombre y la mujer; el respeto a la evolución de las facultades de los niños y las niñas con discapacidad y de su derecho a preservar su identidad. Uno de los conceptos que se estima relevante destacar es el de ajustes razonables, en función de necesidades individuales, en referencia a las adecuaciones particulares que cada persona necesita para su desenvolvimiento y su derecho a ser provista de aquellas. Surge el interrogante de cómo se determina esa necesidad y su razonabilidad, Argentina suscribió la Convención y protocolos facultativos en 2006 y promulgó la ley 26.378 del año 2008 (Dell' Anno, 2012, pp. 14-15). Nuestra Constitución Nacional consagra estas perspectivas de modo genérico en el artículo 16 de su texto.

La Organización Mundial de la Salud establece que, con el fin de mejorar el acceso de las personas con discapacidad a los servicios de salud: orienta y apoya a los Estados Miembros para que aumenten la conciencia con respecto a los problemas de la discapacidad y promueve la inclusión de esta como un componente de las políticas y programas nacionales de salud; facilita la recopilación y divulgación de datos e información acerca de la discapacidad; elabora instrumentos normativos, en particular directrices para fortalecer la asistencia sanitaria; genera capacidad entre los formuladores de políticas y los prestadores de servicios de salud; 
¿Puede Concursar como docente de la Facultad de Derecho de la Universidad DE BUENOS AIRES UNA PERSONA CON DISCAPACIDAD FÍSICA PARA EXPRESARSE?

- Romina Del VAlle ARAMburu CóRdoba (PP. 347-366)

fomenta la ampliación de la rehabilitación en la comunidad; promueve estrategias para velar porque las personas con discapacidad estén enteradas acerca de sus propios problemas de salud y porque el personal sanitario apoye a las personas con discapacidad y proteja sus derechos y dignidad(2).

\section{Normativas de la Universidad Nacional de Buenos Aires y de la Facultad de Derecho. "El derecho de enseñar". Perspectivas de implementación}

De acuerdo con lo visto hasta ahora, en las normas jurídicas nacionales e internacionales no hay lugar a dudas de los derechos que tienen los ciudadanos. Sin embargo, cuando la cuestión se tiene que aplicar al caso particular que se presente, como lo es el caso que en este trabajo se plantea, hay que recurrir a la normativa del ámbito académico. El problema radica en la ausencia de regulaciones normativas en las instituciones educativas universitarias de la Universidad de Buenos Aires. No niega el derecho, pero tampoco habilita los mecanismos para que se efectivice en la práctica. Aquí parece el debate entre el ser y el deber ser o entre lo que dicen las normas constitucionales y la realidad del docente que quiere postularse a un cargo docente con una discapacidad.

En las normas de la Universidad de Buenos Aires y las de la Facultad de Derecho no hay aspectos expresos de carácter normativo que le impidan a una persona con discapacidad física postularse a un concurso docente, cumpliendo con los requisitos de idoneidad y administrativos que se requieren. Si existieran, sería gravemente discriminatorio.

Con relación al tema de las discapacidades, están aquellas que son de nacimiento y las que sobrevienen a posteriori en la vida de los sujetos. En el último caso, los docentes solicitan - cuando es viable- la jubilación por discapacidad; la norma que lo contempla es el artículo 46 del Convenio Colectivo de Trabajadores de las Universidades Nacionales, en su inciso d) referido a la incapacidad. En ella se va a buscar comprobar las lesiones o enfermedades por las cuales hubiese tenido licencia previa y si las mismas son curables o su carácter es irreversible, situación que va a dificultar la continuidad laboral. En ese caso, al docente afectado se le va a designar un profesional médico que dependa de su ámbito laboral. El mismo deberá determinar: "1) índice de incapacidad psicofísica en relación a las leyes previsionales vigentes. 2) determinar, en función a dicho índice, el tipo de funciones que podrá desempeñar sin agravar su estado o el acogimiento a los beneficios jubilatorios. En caso de discordancia se aplicará lo dispuesto en el artículo 47 (...)".

(2) Recuperado de https://www.who.int/es/home/search?query=DISCAPACIDAD\&page=1\&pa gesize $=10 \&$ sortdir $=$ desc $\&$ sort $=$ 
Así lo vemos en la práctica, aunque también puede ocurrir que la persona en cuestión quiera seguir dando clases a pesar de las dificultades que lo limitan; en estos casos hay que analizar cuáles son las posibilidades que el ámbito académico le brinda para poder desempeñar el rol docente.

Lo primero que se debe analizar es, por un lado, las eventuales limitaciones físicas para concursar y también cuáles son las herramientas que le ofrece a quién atraviese esta situación por parte del ámbito académico para que el postulante no quede excluido.

\section{Posición a tomar por parte del ámbito académico}

El primer ámbito académico en el que hay que referenciarse es la Universidad de Buenos Aires y la primera cuestión es recurrir a la normativa vigente. En la Resolución del Consejo Superior No 4362/12 del Reglamento de Concursos de Profesores Regulares no aparece ninguna especificación al respecto de la temática aquí abordada. La misma ha guardado silencio. No es la única: a continuación, se realizará un análisis de otras normas y sus disposiciones, teniendo en cuenta que la Universidad de Buenos Aires establece las normas en las que se basarán las Facultades bajo su órbita. En particular se analiza aquí la Facultad de Derecho.

Si lo analizamos en cuanto a las eventuales limitaciones físicas para concursar, ninguna persona que cumpla con los requisitos de admisión para inscribirse a un concurso docente va a ser vedado de tal posibilidad, aun cuando tuviera una limitación física.

Cuando se plantean cuestiones de discapacidad física se deben analizar los casos en particular y no generalizar, porque cada cuestión debe ser estudiada o atendida dependiendo del caso que se plantee. Los impedimentos físicos a los que me refiero en este trabajo son los que se relacionan con la pérdida de la voz o la pérdida de la capacidad de escribir, indispensables ambas en la labor docente.

"En referencia a las prestaciones en discapacidad tenemos un sistema muy avanzado, pero que para hacerse efectivo depende a veces de la interposición de amparos judiciales. Las prestaciones son costosas, por lo cual entre todos colaboramos y este aporte colectivo debe ser honrado con una gestión responsable de esos recursos, entendiendo que son limitados" (Seda, 2017, p. 139).

Tal como se ha planteado para que un postulante pueda concursar, la Facultad deberá, eventualmente, brindar medios o herramientas para la concreción de tal fin. 
¿Puede Concursar como docente de la Facultad de Derecho de la Universidad DE BUENOS AIRES UNA PERSONA CON DISCAPACIDAD FÍSICA PARA EXPRESARSE?

- Romina Del VAlle ARAMburu CóRdoba (PP. 347-366)

Dependiendo de cuál sea la limitación que pueda existir, la tecnología podrá ser un elemento de importancia en la búsqueda de la igualdad de oportunidades que tenemos las personas.

Siguiendo con esta línea, ¿se puede pensar en el caso de que alguna persona que no haga uso de la palabra pueda - su exposición — ser reemplazada por una computadora que transforme el texto escrito en voz humana? Si la tecnología existe, no habría inconveniente en poder aplicarse. El problema se plantearía en que el ámbito académico no cuente con esa computadora, el programa que requiera y la tecnología complementaria; ello puede suceder por los costos - por ejemploy la afectación del presupuesto anual a estas cuestiones.

También se puede pensar en un aspecto, quizá más económico en costos materiales: la posibilidad de que el docente que se encuentre en esta situación pueda dictar cursos virtuales, a través de diversas modalidades.

En principio, la reglamentación de concursos para la provisión de cargos de profesores regulares tanto de la Universidad de Buenos Aires como de la Facultad de Derecho guardan silencio con respecto a estas posibilidades, así se deprende en lo que vemos a continuación:

1) El Reglamento interno para la provisión de cargos de Profesores regulares titulares, Asociados y adjuntos de la Facultad de Derecho de la Universidad de Buenos Aires, de fecha 14 de abril de 2010, del que surge por invocación la Resolución 6008/09, dictada por el Consejo Directivo de la Facultad de Derecho UBA. En el citado reglamento interno se hace referencia a los requisitos administrativos para la presentación al concurso, designación y actuación del jurado, entrevista personal, prueba de oposición.

2) Por otro lado, el Reglamento General de la Universidad de Buenos Aires número 4362, que surge del expediente 2.259.742/2009, sigue lo dispuesto por la Resolución (CS) 1670/10; aprobó el texto ordenado para la provisión de cargos de profesores regulares titulares, asociados y adjuntos de la Universidad de Buenos Aires de conformidad con las resoluciones del Consejo Superior número 1922/03 y sus modificatorias números 3313/04, 2717/07, $3252 / 07 ; 4118 / 08,4378 / 08$ y 5978/09 respecto al alcance de las prórrogas en las designaciones a los docentes que ejercen funciones como autoridades de la UBA.

3) El Reglamento para la provisión de cargos de Profesores regulares titulares, asociados y adjuntos, basado en la Resolución (CS) Número 1922/2003 y sus modificatorias (CS) números 3313/04; 2717/07, 3252/07, 4118 (desde el $25 / 06 / 08$ ) y 5985/09. En ella se hace referencia a las cuestiones relativas 
a los llamados a concurso. También las condiciones que se requieren para presentarse en un concurso docente. Se hace referencia a los requisitos administrativos y académicos que deben cumplirse para concursar y no se hace mención a ninguno de los aspectos que se intentan tratar en el presente trabajo. Se tratan cuestiones relativas a la designación de los jurados, su actuación, designación de profesores regulares, concursos de renovación y disposiciones generales. El anexo II se refiere a las inhabilitaciones para concursar como las siguientes: el condenado por delito doloso por el lapso que dure la pena, el condenado por delito contra la administración pública mientras dure su sanción, el que fuera inhabilitado para el ejercicio de cargos públicos durante el lapso que dure la inhabilitación, el sancionado por un juicio académico, por mencionar algunos de ellos.

4) Resolución del Consejo Directivo de la Facultad de Derecho de la UBA, No 781/18 del 20 de diciembre de 2018, que establece la paridad de género en la designación de docentes para asegurar la igualdad de oportunidades para las mujeres.

5) Reglamento para la provisión y renovación de cargos de profesores regulares en el ámbito de la Universidad de Buenos Aires Resolución 1922/2003; surge la aprobación del Anexo de las Normas de Procedimiento complementarias para la implementación del Sistema integrado de concursos docentes, Resolución 1242/2004, referida a la pre inscripción en la página web de la unidad académica en donde se postulen para concursar.

6) Resolución 3382/2015(CD) de la Facultad de Derecho de la UBA para la provisión de cargos de auxiliares docentes y las Resoluciones (CD) № 4084/16, $5182 / 17$ y 5413/17. Lo encontramos más detalladamente en el Régimen de Carrera Docente. En la estructura de cargos del régimen de carrera docente, en la resolución (CS) № 3667/15, de creación del régimen de la carrera docente en el ámbito de la Facultad de Derecho. Por otro lado, el Reglamento de concursos para la provisión de cargos de auxiliares docentes, Resolución (CS) № 3382/15, referido a la aprobación del Reglamento para la provisión de cargos de auxiliares docentes en la Facultad de Derecho. En la Resolución (CD) No 5635/18 se introducen modificaciones adecuadas a nuevas normativas reformulando artículos del Anexo I de la Resolución (CS) No 3382/15. Por último, la Resolución (CS) № 3481/07 (Anexo II) hace referencia al Programa de formación y actualización docente continua. En todas las normativas especificadas no se ha previsto nada vinculado con el tema aquí propuesto, que es la postulación a un cargo docente por parte de la persona que cumpla con todos los requisitos legales para ello pero cuente con una discapacidad. 
¿Puede Concursar como docente de la Facultad de Derecho de la Universidad DE BUENOS AIRES UNA PERSONA CON DISCAPACIDAD FÍSICA PARA EXPRESARSE?

- Romina del Valle Aramburu Córdoba (PP. 347-366)

De su lectura se desprende que no contempla el cupo laboral para personas con discapacidad que sí existe en la administración pública nacional provincial y municipal. Existe un cupo en cargos estatales para personas con discapacidad, según la ley No 22.431 (publicada en el Boletín Oficial del 20 de marzo de 1981, con las modificaciones de la ley No 25.689, publicada en el Boletín Oficial del 3 de enero de 2003). El artículo 8, refiriéndose al Estado Nacional —entendiéndose por tal los tres poderes que lo constituyen, sus organismos descentralizados o autárquicos, los entes públicos no estatales, las empresas del Estado y las empresas privadas concesionarias de servicios públicos-, establece que: “(...) están obligados a ocupar personas con discapacidad que reúnan condiciones de idoneidad para el cargo en una proporción no inferior al cuatro por ciento (4\%) de la totalidad de su personal y a establecer reservas de puestos de trabajo a ser exclusivamente ocupados por ellas". "El porcentaje determinado en el párrafo anterior será de cumplimiento obligatorio para el personal de planta efectiva, para los contratados cualquiera sea la modalidad de contratación (...)" (Seda, 2017, p. 12).

La ley 24314 de accesibilidad de personas con movilidad reducida, modificatoria de la ley 22.431, consagra herramientas que le van a servir en la práctica a personas con discapacidades y que los Estados deben garantizar su cumplimiento con carácter obligatorio, como "la prioridad de la supresión de barreras físicas en los ámbitos urbanos, arquitectónicos y del transporte que se realicen o en los existentes que remodelen o sustituyan en forma total o parcial sus elementos constitutivos, con el fin de lograr la accesibilidad para las personas con movilidad reducida", establecida en el artículo 1, fijándose un plazo no mayor de tres años a partir de la sanción de la ley (artículo 2), debido a la urgencia que amerita la rapidez con la que deben arbitrarse los medios para que las personas con dificultades no tengan impedimentos de acceso en los edificios o similares, y de un año en lo que respecta a los medios públicos de transporte y condiciones de adecuación; caso contrario, traerá aparejada la cancelación del servicio.

Si bien las cuestiones a tener en cuenta en los accesos a la docencia y a ser planta permanente en la administración pública para contemplar el cupo son diferentes, tal como lo señala el Doctor Juan Seda, "para ganar un concurso para un cargo de profesor hay que demostrar que se es el mejor en una determinada disciplina académica, en un campo del conocimiento científico. En cambio, en los concursos de ingreso para empleados no docentes se requiere acreditar la idoneidad para el ejercicio de ese cargo, las tareas son más homogéneas (...). También vale la pena distinguir entre la estabilidad del empleado público y la periodicidad en los cargos docentes (...)" (2017, p. 14). Ello nos lleva a replantearnos, en cierta medida, más allá de estas diferencias legales y técnicas que son verídicas, la estabilidad del docente que se plantea en los convenios colectivos de trabajo para los docentes universitarios. 
Muy acertadamente el autor hace una clara diferenciación, además de las mencionadas, entre un rango: el de los docentes de universidades nacionales con normas propias y los empleados públicos, de lo que se deduce que no exista aún el cupo por discapacidad para los docentes universitarios que sí existe para los empleados públicos.

Volviendo a la normativa académica de la Universidad Nacional de Buenos Aires y de la Faculta de Derecho que le pertenece, no aparece algún aspecto que indique que pueden acceder teniendo una discapacidad física para concursar; lo que sí está en claro son los requisitos administrativos de inscripción a los concursos docentes universitarios en la medida que se cumplan con los requisitos de la mencionada normativa, tanto administrativos como académicos.

Supongamos que se realizan las postulaciones y llega el momento de la sustanciación del concurso. La pregunta que aparece es, si hay un vacío legal respecto a cómo va a llevarse a cabo la clase de oposición del postulante con dificultades físicas para expresarse por escrito o verbalmente, ¿hay tecnología que se le pueda proporcionar como un medio para que el /la postulante pueda hacerlo sin incurrir en una eventual discriminación? Si la hubiera ¿cuál sería?

Parece un juego de palabras, pero no lo es; ningún ámbito académico está exento de que se le presente una situación como la que estamos planteando.

Si tomamos el caso de aquel que no tiene el don del habla y quiere presentarse a concursar para un cargo docente, el análisis primario que podrá hacerse es: ¿si no puede hablar para que se presenta si no podrá dar clases? No hay nada que le prohíba a un ciudadano a llegar a esta situación, y si lo pensamos en los tiempos que vivimos, en los que prácticamente todas las tecnologías están puestas al servicio de las personas no sería impedimento que alguien no se valiera de ellas para usarlas en su beneficio como lo es el caso que aquí planteo.

Dicho de otra manera, quién es mudo puede inscribirse en un concurso docente, no hay normas que se lo impidan; si hay un vacío legal administrativo del ámbito académico de cuáles son los procederes en ese caso.

Si lo pensáramos desde un punto de vista más práctico, para este caso particular lo más viable es el lenguaje de señas, entonces la Facultad tendría que destinar parte de su presupuesto en afectar - al principio, pocos, pero después pueden ser muchos- traductores del lenguaje de señas para suplir estas ausencias de voz de otras personas, pero tampoco está garantizado en todo momento que se lo requiera - por ejemplo, un concurso- que se cuente con dicho traductor, como tampoco es una garantía que lo vaya a haber en todas las clases. 
Aquí aparece otra cuestión: ¿qué características debe reunir un traductor de lenguaje de señas al momento de traducir lenguaje jurídico? ¿Y si, llegado el caso, el postulante tiene objeciones que hacer al despliegue de su traductor? Podría pensarse que, para ofrecer más garantías, se realice una filmación de todos los lenguajes en juego en el momento de un concurso docente. La cuestión queda planteada desde el que se postula y quiere concursar. La otra cuestión que se genera como objeto de análisis para otro trabajo es el dictado de las clases en el ámbito áulico.

Es bastante compleja la cuestión. Es una herramienta tecnológica básica comparándola con otras que existen en la actualidad.

\section{VII. ¿Es una cuestión que el ámbito académico debe resolver a instancia de parte?}

En principio, tal como se viene planteando la cuestión, apunta a transformarse en un problema. No lo sería si cada ámbito académico tuviera arbitrados mecanismos legales y prácticos al respecto para que nadie quede afuera de una postulación concursal docente. De allí surge la necesidad de que haya herramientas jurídicas desde un principio, antes de que la aparición del conflicto. Dicho de otra manera, en caso de presentarse una situación como la planteada, ya tener arbitrados los mecanismos para solucionar de la mejor manera para las partes la cuestión.

Se evitaría, por ejemplo, que se les presentara de pronto la situación y que deban resolverla de manera apresurada para cumplir con los tiempos administrativos ante un planteo, o, en un caso más extremo, en el judicial, ante la falta o tardanza en la expedición, cuando el postulante pone en conocimiento de la facultad esta dificultad con el fin de que esta arbitre los medios tecnológicos para hacer su clase de oposición.

Todo planteo administrativo lleva tiempos que, en muchos casos, no se condicen con los tiempos reales de las partes en cuestión, y la solución debería ser de la mejor manera posible para que el postulante concurse sin dificultades.

Las soluciones que puedan pretenderse por parte de cada ámbito académico no deberían ser a instancia de parte, sino ya tenerla arbitrada para cuando se presente el caso en particular. Tampoco hay que esperar una orden judicial, porque ocurriría algo similar a lo planteado anteriormente: se resuelve el caso particular y queda irresuelto cualquier caso que se presente después, teniendo en cuenta que, para llegar a la instancia judicial, hay que agotar la vía administrativa y los tiempos no son tan estrechos. 
Las limitaciones que existen en materia educativa relacionadas con temas de discapacidades ocurren en materia de salud

En referencia a las prestaciones en discapacidad tenemos un sistema muy avanzado, pero que para hacerse efectivo depende a veces de la interposición de amparos judiciales. Las prestaciones son costosas, aporte colectivo debe ser honrado con una gestión responsable de esos recursos, entendiendo que son limitados. Es un complejo desafío que requiere de sentido práctico para ofrecer soluciones concretas sin desfinanciar el resto del sistema de salud (Seda, 2017, p. 109).

\section{Cuando la promoción en la teoría y en la práctica de las igualdades pueden generar desigualdades}

Desde el punto de vista teórico y desde el punto de vista práctico, la sociedad coincide que hay que integrar y no excluir a las personas con discapacidad como regla general. El planteo de una regla general no habilita excepciones, porque ella sería la desigualdad, no en perjuicio de la persona con discapacidad sino del que no la tiene.

Cada caso tiene que analizarse particularmente, evaluar ventajas y desventajas de todas las partes involucradas, alumnos/as, autoridades de un ámbito académico, y habría que ver si no se genera otro problema a posteriori, como se dijo antes: el dictado efectivo de las clases después de haber pasado la instancia del concurso y la designación por parte del Consejo Directivo de la Facultad, pero ese sería otro análisis que no forma parte de este trabajo.

Siguiendo con la cuestión de no generar desigualdades, se conoció un caso jurisprudencial en el que "Una persona con discapacidad motora que había obtenido el título de Licenciado en Educación Física en una Universidad Nacional interpuso acción de amparo a fin de que se ordene a esta última inscribirlo en el Profesorado de esa disciplina. Asimismo, solicitó que se lo eximiera de cumplir con los requerimientos físicos previstos para el ingreso o que se realizaran los ajustes razonables de acuerdo con sus posibilidades. El juez hizo lugar a la acción. La Cámara confirmó el decisorio" (fallos: 117:771, CFed. San Martín, sala II, 17/03/2014, "E. P. N. c. Universidad Nacional de La Matanza (UNLAM) s/ amparo Ley 16.986").

Los fundamentos fueron, entre otros, que "la negativa dispuesta en tal sentido es un acto de arbitrariedad e ilegalidad manifiestas, dado que contradice la normativa legal y supralegal, los propios motivos fundadores del ente educativo y los de la educación superior según el concepto de 'universidad' del legislador". 
El derecho a la educación no le puede ser negado a ningún ciudadano/a, pero la cuestión de conflicto puede aparecer cuando el que no tiene discapacidad alguna y que se puede inscribir a ese profesorado tiene exigencias que no las tiene quién fue inscripto por una orden judicial.

Obviamente que las limitaciones físicas son obstáculos insalvables desde lo físico, pero se puede implementar incluso desde lo curricular de otra materia la posibilidad de que haga actividades teóricas, o de la índole que sean, para tratar de que todos los estudiantes reciban el mismo tratamiento; por ese motivo, se le ordenó a la Universidad no solo la inscripción de la persona en cuestión en el Profesorado sino también que los requerimientos físicos se ajusten a las destrezas de las personas con capacidades reducidas. Este análisis desde la óptica de quién se le ha negado la inscripción a una facultad bien puede tener un sentido análogo a quién quiere concursar con problemas de discapacidad como se plantea aquí.

\section{Evitar las prácticas discriminatorias}

Un amparo contra la provincia de Neuquén, presentado por una mujer que padece disminución visual en ambos ojos, provocó que el Ejecutivo se comprometiera a emitir un decreto para que en todos los concursos se respete el cupo mínimo del 4 por ciento para personas discapacitadas.

Se planteó el caso de una mujer que padece una disminución visual de carácter congénito en los ojos. Sin embargo, esta discapacidad no le impidió cursar estudios de perito mercantil con especialidad auxiliar en administración y desempeñarse en empleos en el ámbito privado de manera informal. En septiembre de 2016 y luego de ser dada de baja de una pensión que cobraba por parte de Nación, la demandante presentó su curriculum ante la Subsecretaría de Discapacidad de la provincia, junto a una nota en la que explicaba su compleja y apremiante situación, ante la imposibilidad de dar sustento a su grupo familiar. Debido a la falta de respuesta, el 20 de enero de 2017 presentó una nueva nota solicitando el ingreso laboral, pero tampoco recibió respuestas. Luego, por acción de funcionarios de la Subsecretaría de Discapacidad y el Ministerio de Ciudadanía, se le asignó un subsidio hasta julio de este año.

La mujer no obtuvo respuesta a su solicitud con base en la ley provincial 1634, que en su artículo 8 obliga al Estado a incorporar a personas con discapacidad al empleo público con una proporción de incorporaciones que no debe ser inferior al 4 por ciento de la planta anual.

Virginia Fernández, a cargo de la Defensoría Adjunta de Gestión, en representación de la actora, presentó una acción de amparo en la que se pidió que se 
ordene a la provincia "cumplir en los llamados a concursos externos con el cupo laboral del 4 por ciento destinado a personas con discapacidad".

El 8 de septiembre, en el Juzgado Civil 3 de Neuquén, se realizó una audiencia de conciliación donde se acordó que la provincia se comprometía a emitir un decreto que ordene en todos los llamados a concurso, en todos los organismos de Estado, a hacer referencia a la ley 1634. Lo dice la misma, "Una obligación del Estado". La ley 25.699 establece la obligación de los tres poderes del Estado a ocupar personas con discapacidad que reúnan condiciones de idoneidad para el cargo. La proporción debe ser no inferior al cuatro por ciento de la totalidad de su personal y el Estado debe reservar ese cupo para ellas.

\section{Conclusión}

En el presente trabajo se ha abordado un análisis acerca de las posibilidades que puede tener una persona para concursar en la Facultad de Derecho de la Universidad de Buenos Aires. Así, me he referido a la cuestión fáctica como caso hipotético, a las normas jurídicas nacionales, supranacionales y las normas propias de la Institución académica citada para hallar a la respuesta a la pregunta: ¿Puede concursar como docente de la Facultad de Derecho de la Universidad de Buenos Aires una persona con discapacidad física para expresarse?

Para ello, hay que tener en cuenta el caso de aquel que no puede expresarse, ya sea de manera oral o escrita, factores indispensables además de cumplir con los requisitos administrativos ya académicos para postularse al cargo y aspirar a ganarlo. La inclusión es un derecho que hace a la dignidad de todos /as los seres humanos. Hasta acá parece la cuestión encaminada, pero nos encontramos con una disparidad de normas en el ámbito interno de la Facultad y de la Universidad que no satisfacen una solución viable para el caso de que se plantee una situación como la propuesta en este trabajo, en el sentido de que seguramente una persona hipotética que quiera concursar no será excluida en la medida que cumpla con los requisitos para los aspirantes a los cargos de docentes, sino dependerá de los medios y herramientas que arbitre la facultad para darle la posibilidad a ese concursante que se postuló efectivamente al concurso.

Para que ello no suceda, aquí se ha propuesto la posibilidad de que se arbitren institucionalmente herramientas tecnológicas; para ello el ámbito académico debe estar preparado y si no lo está deberá hacer una inversión en esta clase de recurso, para lo cual es menester una decisión política. Esta cuestión puede estar sujeta a cuestiones burocráticas que retardarían la efectivización de derechos, y dicho retardo lleva al peligro de convertirlo en un derecho vulnerado. Peor sería si no hay herramientas para resolverlo y se debe recurrir a la instancia judicial. 
¿Puede Concursar como docente de la Facultad de Derecho de la Universidad DE BUENOS AIRES UNA PERSONA CON DISCAPACIDAD FÍSICA PARA EXPRESARSE?

- Romina Del VAlle ARAMburu CóRdoba (PP. 347-366)

La vía judicial tampoco soluciona el conflicto. Por un lado, por los tiempos, aunque sea un amparo judicial y, por otro lado, le daría una respuesta a ese litigante, pero ante la posibilidad futura de otras situaciones similares deberán accionar individualmente. También guarda silencio el convenio colectivo.

Es evidente el vacío legal en materia de discapacidad y acceso a los cargos concursales. En el trabajo detallo las cuestiones que están reguladas en relación con los concursos, pero en el caso que atraigo no hay una previsión normativa al respecto. Es necesario, con carácter urgente, se diriman estas cuestiones, tanto en el Consejo Superior de la Universidad de Buenos Aires como, en consonancia, en cada uno de los H.C.D. de la Facultad de Derecho, arbitrando soluciones inclusivas en los ámbitos de la educación superior teniendo en cuenta las diversidades.

\section{Bibliografía}

Dell'Anno, J. (2012). Debates y perspectivas en torno a la discapacidad en América Latina, 1ra Ed. Paraná: Editorial de la Universidad Nacional de Entre Ríos (UNER). Facultad de Trabajo Social.

Mareño Sempertegui, M. (2012). El Saber convencional sobre la discapacidad y sus implicancias en las prácticas. En M. A. Angelino y M. E. Almeida (comps.), Debates y perspectivas en torno a la discapacidad en América Latina. Paraná: Universidad Nacional de Entre Ríos. UNER. Facultad de Trabajo Social.

Organización mundial de la Salud (2020). Concepto de discapacidad. Recuperado de https://www.who.int/es/home/search?query=DISCAPACIDAD\&page=1 \&pagesize $=10 \&$ sortdir $=$ desc\&sort $=$ [Fecha de consulta: 16/08/2020].

ONU (1948). Declaración de los Derechos Humanos. Recuperado de https://www. un.org/es/universal-declaration-human-rights/ [Fecha de consulta: 10/05/2020].

ONU (1982). Normas uniformes sobre igualdad de oportunidades para personas con discapacidad. Recuperado de http://www.defensoria.org.ar/wp-content/ uploads/2017/02/Normas-Uniformes-de-las-Naciones-Unidas-sobre-la-igualdad-de-oportunidades-para-las-personas-con-discapacidad.pdf [Fecha de consulta: 05/06/2020].

Poder Judicial de Neuquén. Ministerio Público de la Defensa (2017). A partir de un Amparo de la Defensa Pública, en todos los concursos se respetará el cupo para las personas discapacitadas. Recuperado de http://www.mpdneuquen.gob. ar/index.php/2-sin-clasificar/683-la-provincia-se-comprometio-a-incluir-elcupo-para-personas-discapacitadas-en-todos-los-concursos-para-acceder-a-laplanta-estatal 
Seda J. A. (2015). La obesidad severa como discapacidad. Ciudad Autónoma de Buenos Aires: La Ley.

Seda, J. A. (2017). En materia de discapacidad: menos retórica y mayor cumplimiento de la ley. Buenos Aires: Abeledo Perrot.

UNESCO (1990). Conferencia Mundial sobre Educación para Todos. Recuperado de https://unesdoc.unesco.org/ark:/48223/pf0000127583_spa [Fecha de consulta: $10 / 05 / 2020]$.

UNESCO (1994). Conferencia Mundial sobre necesidades educativas especiales: acceso y calidad. Recuperado de https://unesdoc.unesco.org/ark:/48223/ pf0000110753_spa [Fecha de consulta: 10/05/2020].

Valiente Noailles, E. (2017). Política integral de cuidados. Cien políticas para la Argentina 2030. Ciudad Autónoma de Buenos Aires: Editorial Carlos Abeledo.

\section{Legislación}

Constitución Nacional Argentina. Buenos Aires: Editorial Zavalía.

Convención sobre los Derechos de las Personas con Discapacidad. Ley 26.378. Recuperado de http://servicios.infoleg.gob.ar/infolegInternet/anexos/140000-144999/141317/norma.htm [Fecha de consulta: 05/05/2020].

Ley No 22.431. Boletín Oficial de la República Argentina, Buenos Aires, 16/03/1981. Recuperado de http://www.saij.gob.ar (documento: LNS0002803) [Fecha de consulta: 05/06/2020].

Ley No 24.314. Boletín Oficial de la República Argentina, Buenos Aires, 15/03/1994. Recuperado de http://www.saij.gob.ar (documento: LNN0028645) [Fecha de consulta: 10/06/2020].

Ley No 26.285. Boletín Oficial de la República Argentina, Buenos Aires, 15/08/2007. Recuperado de http://www.saij.gob.ar (documento: LNN0029613) [Fecha de consulta: 10/06/2020].

Ley No 26.653, Boletín Oficial de la República Argentina, Buenos Aires, 30/11/2010. Recuperado de http://www.saij.gob.ar (documento: LNS0005653). [Fecha de consulta: 15/06/2020].

Ley No 26.858. Boletín Oficial de la República Argentina, Buenos Aires, 14/06/2013. Derecho de Acceso, deambulación y permanencia. Personas con discapacidad acompañadas por Perro Guía o de Asistencia. Recuperado de http:// www.saij.gob.ar (documento: LNS0005846) [Fecha de consulta: 11/06/2020]. 
Ley No 64. Boletín Oficial de la Ciudad de Buenos Aires, Buenos Aires, 20/08/1998. Baños con sistema Braille o silueta en relieve en locales públicos. Recuperado de http://www.saij.gob.ar (documento: LPX0000064) [Fecha de consulta: 11/06/2020].

Ley No 161. Boletín Oficial de la Ciudad de Buenos Aires, Buenos Aires, 25/02/1999. Puertas de Cabina y Rellano de Ascensores. Recuperado de http:// www.saij.gob.ar (documento: LPX0000161). [Fecha de consulta: 15/06/2020].

Ley No 429. Boletín Oficial de la Ciudad de Buenos Aires, Buenos Aires, 29/06/2000. Acceso de perros guías al transporte público, espacios públicos o de acceso público. Recuperado de http://www.saij.gob.ar (documento: LPX0000429) [Fecha de consulta: 11/06/2020].

Ley No 962. Boletín Oficial de la Ciudad de Buenos Aires, Buenos Aires, 05/12/2002. Accesibilidad física para todos. Modificación de la Ley No 161. Recuperado de http://www.saij.gob.ar (documento: LPX0000962) [Fecha de consulta: 15/06/2020].

Decreto No 914/1997. Boletín Oficial de la República Argentina, Buenos Aires, 11/09/1997. Reglamentación de los artículos 20, 21 y 22 de la Ley No 22.431 modificados por su similar № 24.314 (suspensión de barreras). Recuperado de http://www.saij.gob.ar (documento: DN19970000914).

\section{Jurisprudencia}

Cámara Federal de San Martín, Sala II, 17/03/2014, "E. P. N. c. Universidad Nacional de La Matanza (UNLAM) s/ amparo Ley 16.986". Recuperado de http:// www.saij.gob.ar (documento: FA13630001) [Fecha de consulta: 28/03/2020].

Fallos: 16:118; 123:106; 124:122. Recuperado de https://www.mpf.gob.ar/ dgdh/files/2016/06/Cuadernillo-2-Igualdad-y-noDiscriminaci\%C3\%B3n.pdf [Fecha de consulta: 20/05/2020].

\section{Resoluciones}

Reglamento interno para la provisión de cargos docentes, Facultad de Derecho, Universidad de Buenos Aires. Recuperado de http://www.derecho.uba.ar/ academica/concursos_docentes/2010_reglamento-interno-concursos-docentes. pdf [Fecha de consulta: 18/01/2020].

Reglamento para la provisión de cargos de Profesores regulares titulares, asociados y adjuntos, basado en la Resolución (CS) Número 1922/2003 y sus modificatorias (CS) números 3313/04; 2717/07, 3252/07, 4118 (desde el 25/06/08) 
y 5985/09 y Anexo II de la Universidad de Buenos Aires. Recuperado de http:// www.derecho.uba.ar/academica/concursos_docentes/reglamento_res_cd-4362. pdf. [Fecha de consulta: 18/01/2020].

Resolución del Consejo Directivo de la Facultad de Derecho de la UBA. Recuperado de http://www.derecho.uba.ar/academica/concursos_docentes/pdf/rescd-781-18-jurado-diversidad-de-genero.pdf. [Fecha de consulta: 18/01/2020].

Reglamento para la provisión y renovación de cargos de profesores regulares en el ámbito de la Universidad de Buenos Aires Resolución 1922/2003 surge la aprobación del Anexo de las Normas de Procedimiento complementarias para la implementación del "Sistema integrado de concursos docentes" Resolución 1242/2004. Recuperado de http://www.derecho.uba.ar/academica/concursos_ docentes/pdf/res_1242-04-preinscripcion.pdf. [Fecha de consulta: 18/01/2020].

Estructura de cargos del régimen de carrera docente. Resolución (CS) № 3667/15. Recuperado de http://www.derecho.uba.ar/academica/carrdocente/regimen. php [Fecha de consulta: 08/01/2020].

Reglamento de concursos para la provisión de cargos de auxiliares docentes. Recuperado de http://www.derecho.uba.ar/academica/carrdocente/regimen.php. [Fecha de consulta: 18/01/2020].

Resolución (CS) No 3382/15. Recuperado de http://www.derecho.uba.ar/academica/carrdocente/regimen.php [Fecha de consulta: 18/01/2020].

Resolución (CD) No 5635/18. Recuperado de http://www.derecho.uba.ar/academica/carrdocente/regimen.php [Fecha de consulta: 18/01/2020].

Programa de formación y actualización docente continua. Resolución (CS) No 3481/07 (Anexo II). Recuperado de http://www.derecho.uba.ar/academica/ carrdocente/regimen.php. [Fecha de consulta: 18/01/2020].

Convenios Colectivos de Docentes de las Universidades Nacionales. Recuperado de https://conadu.org.ar/wp-content/uploads/CCT-completo.pdf [Fecha de consulta: 18/01/2020].

Fecha de recepción: 17-03-2021

Fecha de aceptación: 05-09-2021 